tances from the kidney pelvis. Other factors being constant, it seems that the length of the hydroureter from the uretero-pelvic junction varies directly with the duration of the terminal overload.

\title{
$63(995)$
}

\section{The resistance to mechanical injury of the erythrocytes of different} species.

\section{By Peyton Rous and J. R. Turner.}

\section{[From the Laboratories of the Rockefeller Institute for Medical Research.]}

As the first step in an analysis of the conditions under which red cells will survive in vitro, we have studied the effects of handling them, as in washing. That this may entail injury is suggested by the work of Meltzer, ${ }^{1}$ who noted that a few minutes' shaking of whole blood hastens considerably the disintegration of the erythrocytes.

Our experiments show that in citrated plasma the red cells of the dog, rabbit, sheep and man withstand well the handling incident to ordinary washing. They may be centrifugalized and suspended again and again without hemolysis. The case is entirely different when cells in salt solution are repeatedly washed and suspended. Except in the case of human cells this entails marked injury, which expresses itself either in an immediate slight hemolysis, or more often in a shortening of the time during which the cells remain intact.

The protective action of plasma, even when dilute, is well shown when washed cells are suspended in it and in isotonic salt solution, and shaken. Hemolysis occurs much sooner in the salt solution. The experiments have brought out striking differences in the fragility of the red cells of different species-and, to a less degree, of different individuals. Dog cells shaken in the salt solution undergo a marked and almost immediate hemolysis. Rabbit corpuscles are somewhat less sensitive, and sheep corpuscles even less so, while the red cells of man are markedly

1 Meltzer, S. J., Johns Hopkins Hosp. Bull., I900, IX, I34. 
resistant, though in their case, too, the protective action of plasma can be demonstrated.

The resistance of the red cells to mechanical injury has little if any relation to their resistance to hypotonic salt solution. For example, the corpuscles of the dog, though much more easily destroyed by shaking than those of the sheep, will withstand a hypotonic salt solution in which the laking of sheep cells is pronounced. With the bloods of different individuals of the same species a similar lack of parallelism in the two resistances has been noted.

\section{$64(996)$}

The protection of fragile erythrocytes against mechanical injury.

By Peyton Rous and J. R. Turner.

[From the Laboratories of the Rockefeller Institute for Medical Research.]

In the preceding paper the fact has been stated that erythrocytes handled in salt solution undergo an injury which does not take place when they are in plasma. This has suggested tests of various substances for a protective action. We have found that the addition to Ringer's solution of gelatin in very small quantity $-\frac{1}{8}$ of I per cent.-protects the red cells completely, and that their prolonged sojourn in it is no more harmful than in plain Ringer's. Dog corpuscles which break down almost at once when shaken in Ringer's solution, resist prolonged shaking when in the gelatin-Ringer's. Corpuscles of the dog, rabbit and sheep, washed in this fluid and placed in ordinary Ringer's, remain intact much longer than when washed in the latter. Dog erythrocytes may keep several days, whereas when washed in plain Ringer's solution they break down within a few hours. Only in the case of human red cells does the protection afforded by gelatin seem unnecessary during washing. These cells last quite as well when handled in plain Ringer's.

That the erythrocytes of certain species differ markedly as regards the time they remain intact when washed and placed in isotonic salt solution, is well known. The experiments with the 